\title{
Core or Cusps: The Central Dark Matter Profile of a Strong Lensing Cluster with a Bright Central Image at Redshift 1
}

Thomas E. Collett ${ }^{1}$, Elizabeth Buckley-Geer ${ }^{2}$, Huan Lin $^{2}$, David Bacon ${ }^{1}$, Robert C. Nichol ${ }^{1}$, Brian Nord ${ }^{2}$, Xan Morice-Atkinson ${ }^{1}$, Adam Amara ${ }^{3}$, Simon Birrer ${ }^{3,4}$, Nikolay Kuropatkin ${ }^{2}$, Anupreeta More ${ }^{5}$, Casey Papovich ${ }^{6}$, Kathy K. Romer ${ }^{7}$, Nicolas Tessore ${ }^{8}$,

Tim M. C. Abbott ${ }^{9}$, Sahar Allam ${ }^{2}$, James Annis ${ }^{2}$, Aurlien Benoit-Lévy ${ }^{10,11,12}$, David Brooks ${ }^{11}$, David L. Burke ${ }^{13,14}$,

Matias Carrasco Kind ${ }^{15,16}$, Francisco Javier J. Castander ${ }^{17}$, Chris B. D’Andrea ${ }^{18}$, Luiz N. da Costa ${ }^{19,20}$, Shantanu Desai ${ }^{21}$,

H. Thomas Diehl ${ }^{2}$, Peter Doel ${ }^{11}$, Tim F. Eifler ${ }^{22,23}$, Brenna Flaugher ${ }^{2}$, Josh Frieman ${ }^{2,24}$, David W. Gerdes ${ }^{25,26}$,

Daniel A. Goldstein ${ }^{27,28}$, Daniel Gruen ${ }^{13,14}$, Julia Gschwend ${ }^{19,20}$, Gaston Gutierrez ${ }^{2}$, David J. James ${ }^{9,29}$, Kyler Kuehn ${ }^{30}$, Steve Kuhlmann ${ }^{31}$, Ofer Lahav ${ }^{11}$, Ting S. Li ${ }^{2,6}$, Marcos Lima ${ }^{19,32}$, Marcio A. G. Maia ${ }^{19,20}$, Marisa March ${ }^{18}$, Jennifer L. Marshall ${ }^{6}$, Paul Martini ${ }^{33,34}$, Peter Melchior ${ }^{35}$, Ramon Miquel ${ }^{36,37}$, Andrs A. Plazas ${ }^{23}$, Eli S. Rykoff ${ }^{13,14}$, Eusebio Sanchez ${ }^{38}$, Vic Scarpine ${ }^{2}$, Rafe Schindler ${ }^{14}$, Michael Schubnell ${ }^{26}$, Ignacio Sevilla-Noarbe ${ }^{38}$, Mathew Smith ${ }^{39}$, Flavia Sobreira ${ }^{19,40}$, Eric Suchyta ${ }^{41}$, Molly E. C. Swanson ${ }^{16}$, Gregory Tarle ${ }^{26}$, Douglas L. Tucker ${ }^{2}$, and Alistair R. Walker ${ }^{9}$

Institute of Cosmology \& Gravitation, University of Portsmouth, Portsmouth PO1 3FX, UK

${ }^{2}$ Fermi National Accelerator Laboratory, P.O. Box 500, Batavia, IL 60510, USA

${ }^{3}$ Department of Physics, ETH Zurich, Wolfgang-Pauli-Strasse 16, CH-8093 Zurich, Switzerland

${ }^{4}$ Department of Physics and Astronomy, UCLA, PAB, 430 Portola Plaza, Box 951547, Los Angeles, CA 90095-1547, USA

${ }^{5}$ Kavli IPMU (WPI), UTIAS, The University of Tokyo, Kashiwa, Chiba 277-8583, Japan

${ }^{6}$ George P. and Cynthia Woods Mitchell Institute for Fundamental Physics and Astronomy, and Department of Physics and Astronomy, Texas A\&M University, College Station, TX 77843, USA

${ }^{7}$ Department of Physics and Astronomy, Pevensey Building, University of Sussex, Brighton BN1 9QH, UK

8 Jodrell Bank Center for Astrophysics, School of Physics and Astronomy, University of Manchester, Oxford Road, Manchester M13 9PL, UK

${ }^{9}$ Cerro Tololo Inter-American Observatory, National Optical Astronomy Observatory, Casilla 603, La Serena, Chile

${ }^{10}$ CNRS, UMR 7095, Institut d'Astrophysique de Paris, F-75014, Paris, France

${ }^{11}$ Department of Physics \& Astronomy, University College London, Gower Street, London WC1E 6BT, UK

${ }^{12}$ Sorbonne Universités, UPMC Univ. Paris 06, UMR 7095, Institut d'Astrophysique de Paris, F-75014, Paris, France

${ }^{13}$ Kavli Institute for Particle Astrophysics \& Cosmology, P.O. Box 2450, Stanford University, Stanford, CA 94305, USA

${ }^{14}$ SLAC National Accelerator Laboratory, Menlo Park, CA 94025, USA

${ }_{16}^{15}$ Department of Astronomy, University of Illinois, 1002 W. Green Street, Urbana, IL 61801, USA

${ }^{16}$ National Center for Supercomputing Applications, 1205 West Clark St., Urbana, IL 61801, USA

${ }^{17}$ Institut de Ciències de l'Espai, IEEC-CSIC, Campus UAB, Carrer de Can Magrans, s/n, E-08193 Bellaterra, Barcelona, Spain

${ }^{18}$ Department of Physics and Astronomy, University of Pennsylvania, Philadelphia, PA 19104, USA

${ }^{19}$ Laboratório Interinstitucional de e-Astronomia-LIneA, Rua Gal. José Cristino 77, Rio de Janeiro, RJ-20921-400, Brazil

${ }^{20}$ Observatório Nacional, Rua Gal. José Cristino 77, Rio de Janeiro, RJ-20921-400, Brazil

${ }^{21}$ Department of Physics, IIT Hyderabad, Kandi, Telangana 502285, India

${ }^{22}$ Department of Physics, California Institute of Technology, Pasadena, CA 91125 , USA

${ }^{23}$ Jet Propulsion Laboratory, California Institute of Technology, 4800 Oak Grove Dr., Pasadena, CA 91109, USA

${ }^{24}$ Kavli Institute for Cosmological Physics, University of Chicago, Chicago, IL 60637, USA

25 Department of Astronomy, University of Michigan, Ann Arbor, MI 48109, USA

${ }^{26}$ Department of Physics, University of Michigan, Ann Arbor, MI 48109, USA

${ }^{27}$ Department of Astronomy, University of California, Berkeley, 501 Campbell Hall, Berkeley, CA 94720, USA

${ }^{28}$ Lawrence Berkeley National Laboratory, 1 Cyclotron Road, Berkeley, CA 94720, USA

${ }^{29}$ Astronomy Department, University of Washington, Box 351580, Seattle, WA 98195, USA

${ }^{30}$ Australian Astronomical Observatory, North Ryde, NSW 2113, Australia

${ }^{31}$ Argonne National Laboratory, 9700 South Cass Avenue, Lemont, IL 60439, USA

32 Departamento de Física Matemática, Instituto de Física, Universidade de São Paulo, CP 66318, CEP 05314-970, São Paulo, SP, Brazil

${ }^{33}$ Center for Cosmology and Astro-Particle Physics, The Ohio State University, Columbus, OH 43210, USA

${ }^{34}$ Department of Astronomy, The Ohio State University, Columbus, OH 43210, USA

${ }^{35}$ Department of Astrophysical Sciences, Princeton University, Peyton Hall, Princeton, NJ 08544, USA

${ }^{36}$ Institució Catalana de Recerca i Estudis Avançats, E-08010 Barcelona, Spain

${ }^{37}$ Institut de Física d'Altes Energies (IFAE), The Barcelona Institute of Science and Technology, Campus UAB, E-08193 Bellaterra (Barcelona) Spain

${ }^{38}$ Centro de Investigaciones Energéticas, Medioambientales y Tecnológicas (CIEMAT), Madrid, Spain

${ }^{39}$ School of Physics and Astronomy, University of Southampton, Southampton SO17 1BJ, UK

${ }^{40}$ Instituto de Física Gleb Wataghin, Universidade Estadual de Campinas, 13083-859, Campinas, SP, Brazil

${ }^{41}$ Computer Science and Mathematics Division, Oak Ridge National Laboratory, Oak Ridge, TN 37831, USA

Received 2017 March 24; revised 2017 May 22; accepted 2017 May 30; published 2017 July 14

\begin{abstract}
We report on SPT-CLJ2011-5228, a giant system of arcs created by a cluster at $z=1.06$. The arc system is notable for the presence of a bright central image. The source is a Lyman break galaxy at $z_{s}=2.39$ and the mass enclosed within the Einstein ring of radius 14 arcsec is $\sim 10^{14.2} M_{\odot}$. We perform a full reconstruction of the light profile of the lensed images to precisely infer the parameters of the mass distribution. The brightness of the central image demands that the central total density profile of the lens be shallow. By fitting the dark matter as a generalized Navarro-Frenk-White profile-with a free parameter for the inner density slope-we find that the break radius is $270_{-76}^{+48} \mathrm{kpc}$, and that the inner density falls with radius to the power $-0.38 \pm 0.04$ at $68 \%$ confidence. Such a shallow profile is in strong tension with our understanding of relaxed cold dark matter halos; dark matter-only
\end{abstract}


simulations predict that the inner density should fall as $r^{-1}$. The tension can be alleviated if this cluster is in fact a merger; a two-halo model can also reconstruct the data, with both clumps (density varying as $r^{-0.8}$ and $r^{-1.0}$ ) much more consistent with predictions from dark matter-only simulations. At the resolution of our Dark Energy Survey imaging, we are unable to choose between these two models, but we make predictions for forthcoming Hubble Space Telescope imaging that will decisively distinguish between them.

Key words: dark matter - galaxies: clusters: individual (SPT-CLJ2011-5228) - galaxies: halos gravitational lensing: strong

\section{Introduction}

Cosmological cold dark matter (CDM) simulations suggest a model of hierarchical structure formation that produces a cuspy dark matter (DM) profile. Navarro et al. (1996) found that the halo profiles are universal and well fit by the form

$$
\rho_{\mathrm{NFW}}(r)=\frac{\rho_{0}}{r\left(r_{s}+r\right)^{2}} .
$$

The mass of a Navarro-Frenk-White (NFW) halo and the scale radius are the only free parameters, and CDM simulations show that these parameters are connected - with some scatter-by a mass-concentration relation (Neto et al. 2007; Duffy et al. 2010). However, baryonic physics complicates this simple CDM picture (Gnedin et al. 2004): gas can radiatively cool and contract, dragging DM inwards and increasing the central density, but supernovae, active galactic nuclei, and dynamical heating can inject energy into the interstellar medium, causing the halo to expand (Abadi et al. 2010; Teyssier et al. 2011; Laporte et al. 2012; Pontzen \& Governato 2012). Halos will also deviate from NFW if the dark matter is self-interacting or if it has a large de Broglie wavelength. In these cosmologies the dark matter self-interaction creates a pressure that leads to the formation of central cores rather than high-density cusps (Vogelsberger et al. 2012; Peter et al. 2013; Rocha et al. 2013). The central density profiles of halos are therefore sensitive probes of both baryonic feedback effects and the CDM paradigm.

Observationally, it has been shown that NFW profiles do not reproduce the central regions of halos over a range of masses. On the scales of dwarf galaxies there is evidence for central dark matter cores (de Blok et al. 2001; Amorisco et al. 2013) derived from kinematic constraints, but recent simulations have cast doubt on the interpretation of these data (Pineda et al. 2017). On the scale of elliptical galaxies there is evidence for cuspy dark matter profiles that are much steeper than NFW. Sonnenfeld et al. (2012) find $\rho \sim r^{-1.7}$ for an individual strong lensing galaxy with multiple background sources, and Grillo (2012) found that the average inner DM slope of 38 strong lenses is close to isothermal although these results depend on the assumed stellar mass-to-light ratio.

On the scale of galaxy clusters the central profiles are often seen to be flatter than NFW. Sand et al. (2004) found that $\rho \sim r^{-0.5}$ for a sample of six strong lensing clusters. Work by Newman et al. (2013a) combining stellar kinematics with strong and weak lensing found that the total mass profile in their clusters was consistent with NFW. However, because the total mass profile in those clusters is dominated by the baryonic content of the brightest cluster galaxy (BCG) they inferred that the dark matter profile was significantly shallower than NFW (Newman et al. 2013b). However, either a shallow central dark matter cusp or a cored NFW profile was able to reconstruct the data. Recently Oldham \& Auger (2016) showed that the dark matter halo of M87 has a $19 \mathrm{kpc}$ core although the core size is degenerate with orbital anisotropy.

The primary difficulty of previous work on clusters has been the presence of a massive galaxy that dominates the total mass profile at the center of the cluster. Inferences on the dark halo are therefore only as robust as the subtraction of the stellar component.

In this work we present imaging, spectroscopy, and modeling of SPT-CLJ2011-5228 (hereafter J2011). J2011 is a cluster of mass $M_{500}=(2.25 \pm 0.89) \times 10^{14} h_{70}^{-1} M_{\odot}$ (Reichardt et al. 2013) at $z=1.064$ acting as a strong gravitational lens with an Einstein radius of $14.01 \pm 0.06$ arcsec (Figure 1). The arc system is notable for the presence of a bright central image. Central images are typically highly demagnified since in dense regions a small perturbation to the impact parameter of a light ray will produce a large change in the deflection angle. To date, very few true central images are known (Colley et al. 1996; Winn et al. 2004; Inada et al. 2005; Sharon et al. 2012), and they are typically highly demagnified. The presence of a bright central image in J2011 is therefore an exciting discovery and opens up the possibility to precisely constrain the central dark matter profile of this cluster.

In Section 2 of this paper we present our observations of J2011 using the Dark Energy Camera on the $4 \mathrm{~m}$ Victor M. Blanco telescope and the GMOS spectrograph on the $8 \mathrm{~m}$ Gemini South telescope. In Section 3, we describe our modeling approach for this lens and present the result of two models for the dark matter distribution in the lens: a one-halo model representing a relaxed cluster scenario in Section 3.1 and a twohalo model representing a merging scenario in Section 4. We provide interpretation of the results in Section 5 and conclude in Section 6. Unless otherwise stated, coordinates are measured west, north relative to 20:11:10.611, $-52: 28: 40.12$ (J2000) with a pixel size of 0.263 arcsec. Throughout this work we assume a flat $\Lambda \mathrm{CDM}$ cosmology with $H_{0}=67.8 \mathrm{~km} \mathrm{~s}^{-1} \mathrm{Mpc}^{-1}$ and $\Omega_{\mathrm{M}}=0.308$ (Planck Collaboration et al. 2016).

\section{Discovery of a Giant Arc System in DES and SPT Data}

J2011 was first reported as one of the 224 galaxy clusters detected in the first $720 \mathrm{deg}^{2}$ of the South Pole Telescope (SPT) Sunyaev-Zel'dovich (SZ) survey. The SPT data selection is described in Reichardt et al. (2013). The cluster has a reported mass of $M_{500}=(2.59 \pm 0.73) \times 10^{14} h_{70}^{-1} M_{\odot}$ with a detection significance of 4.58 (Bleem et al. 2015). The SPT collaboration carried out follow-up optical and NIR imaging of these clusters using various telescopes and instruments as described in Song et al. (2012). In Table 3 of Song et al. (2012) this cluster is noted as having a strong lensing arc. This area of the SPT footprint was imaged as part of the Year 1 (Y1) observations (Diehl et al. 2014; Flaugher et al. 2015) of the Dark Energy Survey (DES, Flaugher 2005). The DES subsequently rediscovered the system in a targeted visual search of all known SPT clusters in the Y1 


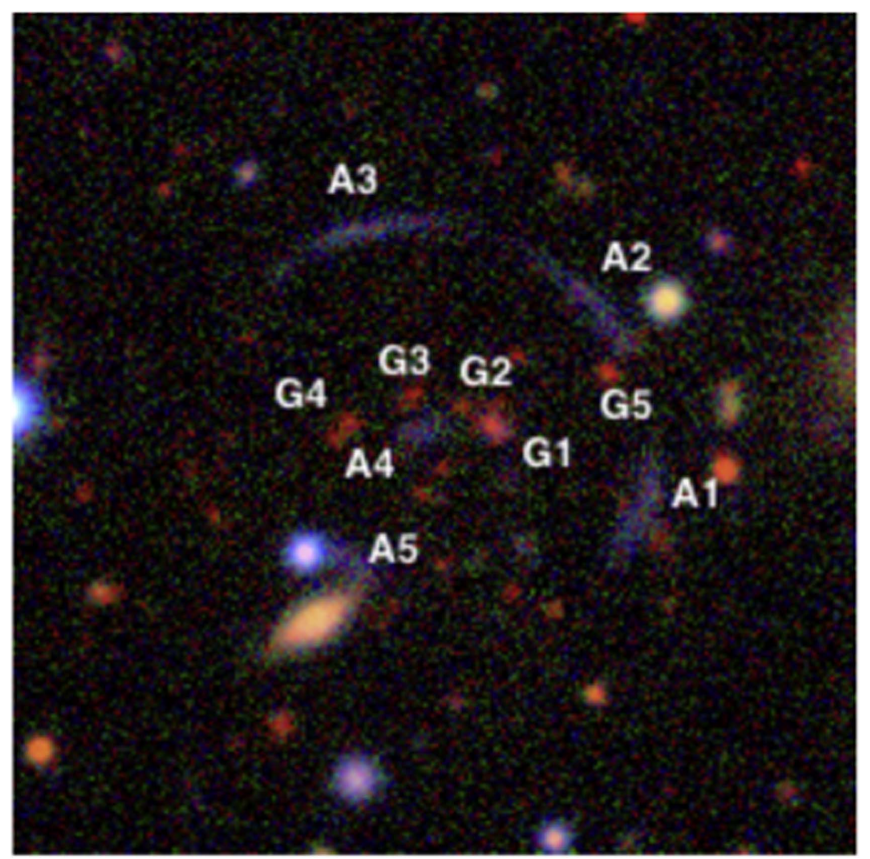

Figure 1. Pseudo-color gri composite image of the lens J2011, taken from the first three years of operation of the Dark Energy Survey. The image is 1 arcminute on a side.

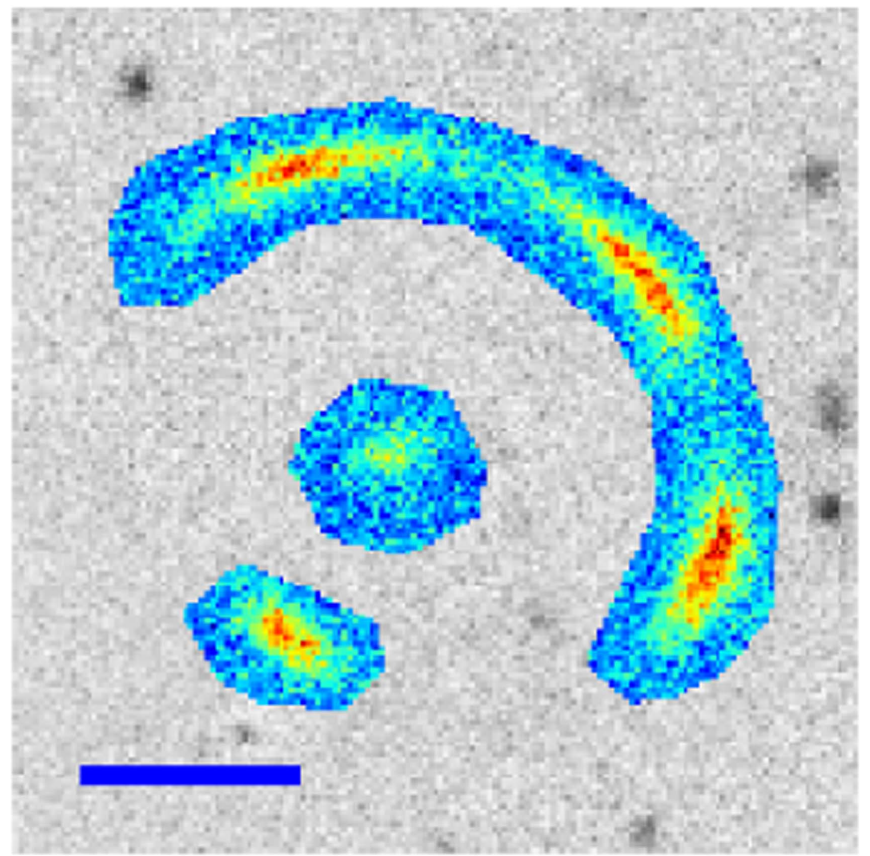

Figure 2. $g$-band image of the arcs and central image, after subtracting foregrounds. Only the colored pixels are included in the lens modeling of Section 3. The blue bar shows a scale of 10 arcsec.

footprint and separately in a visual search of red sources with multiple neighbors (H. T. Diehl 2017, in preparation). The visual scans were performed on false-color PNG images, which are made by combining $g, r, i$ coaddition tiles into color images (L. N. da Costa 2016, private communication).

\subsection{DES Imaging}

The lens modeling requires as input an image of the lensed arcs, a sigma image, and a point-spread function (PSF) for each band.
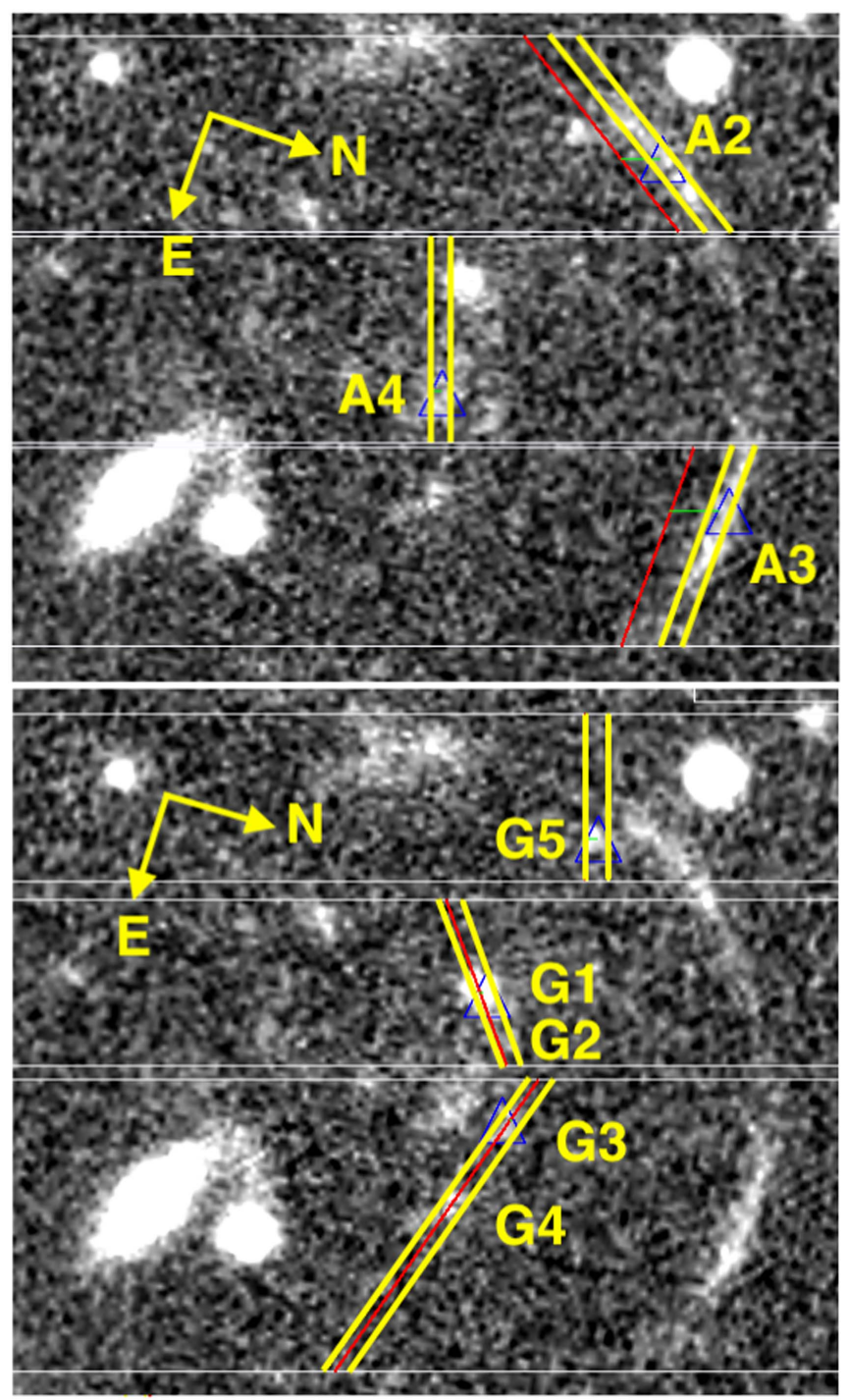

Figure 3. The Gemini GMOS masks for the spectroscopic observations. Top: source mask. Bottom: cluster mask.

We use imaging from the first three years of DES to provide these data. The DESDM image processing pipeline (summarized in Balbinot et al. 2015 and described in detail in Sevilla et al. 2011; Desai et al. 2012; Mohr et al. 2012) carries out image coaddition for each band and creates a sigma image, which is the total uncertainty on each pixel of the coadd image due to noise. The DESDM pipeline also fits a model of the PSF using PSFEX (Bertin \& Arnouts 1996; Bertin 2011). The PSF model is used to generate a model star at the location of the cluster. The default DESDM sigma image only includes the noise from the sky photons and not the photons contributing to the objects in the image. We therefore modified and re-ran the image processing pipeline to generate a sigma image that also includes shot noise from the objects.

With our data, the lensed arc is brightest in the $g$-band, where there is negligible contribution to the flux from the cluster members, since the Balmer break lies redward of the $g$-band filter. There is, however, $g$-band emission close to arcs associated with two foreground stars (close to A2 and A5), a foreground galaxy (close to A5), plus emission from the BCG (which is close to the central image A4) (potentially produced by an active galactic 
Table 1

Spectroscopic Observation Log

\begin{tabular}{|c|c|c|c|c|c|}
\hline Object & UT Date & $\begin{array}{l}\text { Telescope- } \\
\text { Instrument }\end{array}$ & Grating & $\begin{array}{l}\text { Total Integration } \\
\text { (hr) }\end{array}$ & $\begin{array}{l}\text { Seeing } \\
(\operatorname{arcsec})\end{array}$ \\
\hline$\overline{\text { Arcs }}$ & 2014 Oct 20,24 & Gemini-GMOS & $\mathrm{B} 600$ & 3 & $0.86,0.66$ \\
\hline Arcs & 2015 Jul 17, 19 & Gemini-GMOS & $\mathrm{R} 150$ & 0.93 & $0.9,1.06$ \\
\hline Lens & 2014 Oct 24 & Gemini-GMOS & $\mathrm{R} 150$ & 1 & 0.66 \\
\hline
\end{tabular}

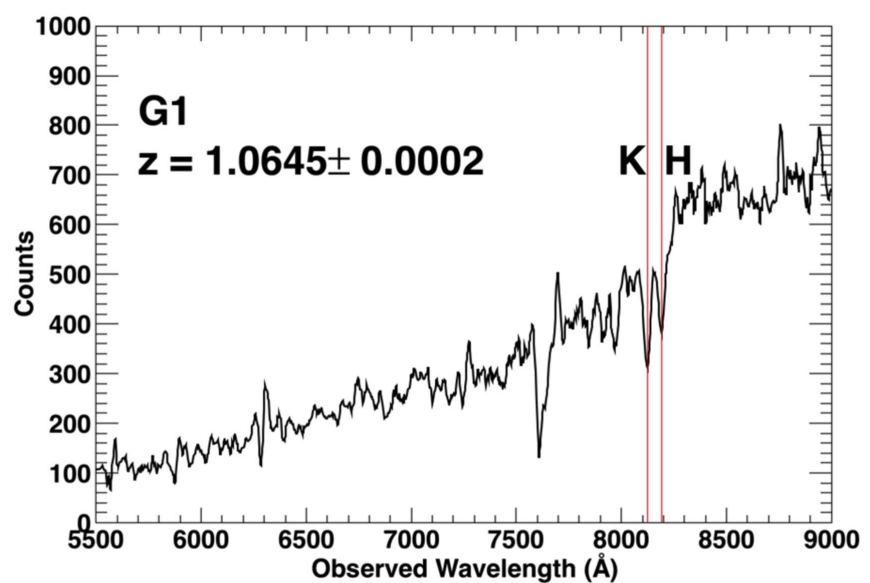

Figure 4. The extracted un-fluxed $1 \mathrm{D}$ spectra for the BCG (G1). The $\mathrm{Ca} \mathrm{H}$ and $\mathrm{K}$ absorption lines are indicated by the red lines.

nucleus). These foregrounds are fitted with point sources for the stars, and Sérsic profiles for the BCG and foreground galaxy. To ensure that flux from the arcs is not attributed to the foregrounds, we simultaneously model the foregrounds and arcs using an elliptical power-law density profile for the lens (Barkana 1998) and a parametric source with three Sérsic components. ${ }^{42}$ This lens model is then optimized and the $g$-band foregrounds subtracted. We use the foreground-subtracted $g$-band data for our lens modeling. The foreground-subtracted $g$-band data are shown in Figure 2; it is these data that we use for the lens modeling in Section 3 .

\subsection{Gemini Spectroscopy of J2011}

We obtained spectroscopic follow-up observations with the Gemini Multi-Object Spectrograph (GMOS, Hook et al. 2004) on the Gemini South Telescope, as part of the Gemini Large and Long Program GS-2014B-LP-5. ${ }^{43}$

We targeted four of the red galaxies, labelled G1-G4 in Figure 1, in the center of the cluster including the BCG (labelled G1) along with another smaller galaxy close to arc A2 (labelled G5) and the arc features labelled A2-A4 using the multi-object mode on GMOS. We created two masks, one for the galaxies and the other for the arc features. The slits were $1^{\prime \prime}$ in width and of varying length in order to accommodate both the object and an amount of sky sufficient to perform reliable background subtraction. For some of the objects, we tilted the slits to maximize the captured flux. The two masks are shown in Figure 3.

\footnotetext{
42 The power-law lens model fits the outer arcs well but does not reproduce the central image.

43 http://www.gemini.edu/?q=node/12238\#Buckley
}

We observed the galaxies and arcs using two configurations with GMOS to cover the full wavelength range from $3250-10000 \AA$. We use the R150 grating in conjunction with the GG455 filter in order to obtain spectra with wavelength coverage $\sim 4500-10000 \AA$. If the source is a late-type emission line galaxy, this would allow us, in most cases, to detect [O II] 3727 to $z \sim 1.7, \mathrm{H} \beta$ to $z \sim 1.0$, and $\mathrm{Ly} \alpha$ in the range $z \sim 2.7-7.2$. We use the B600 grating to obtain spectral coverage of $3250-6250 \AA$, which would allow us to detect sources with $z>2.0$ that emit $\operatorname{Ly} \alpha$. The observations are summarized in Table 1. The seeing is taken from the Gemini data quality assessment.

An observing sequence consisted of a pair of exposures, followed by a flat field taken with a quartz-halogen lamp and a calibration spectrum taken with a CuAr arc lamp. We then dithered to a different central wavelength to cover the gap between the CCDs and took a CuAr spectrum, followed by the flat-field exposure, and then two more exposures. Exposure times were either $900 \mathrm{~s}$ or $840 \mathrm{~s}$. Dividing the integration time into multiple exposures facilitates the removal of cosmic rays. The data were binned $2 \times 2$, giving effective dispersions of 0.1 and $0.386 \mathrm{~nm} \mathrm{pixel}{ }^{-1}$ for the B600 and R150 gratings, respectively.

We used the Gemini IRAF package v2.16 ${ }^{44}$ to reduce all exposures. In each system, for each wavelength dither, we first process the flat field using the task gsflat (this includes subtraction of the bias). Each science exposure in a single dither is then reduced with gsreduce (using the previously processed flat fields), and then the two exposures are combined with gemcombine. Wavelength calibration and transformation are performed on each dither (using the tasks gswavelength and gstransform) before the pairs of dithers are coadded on a common wavelength scale to eliminate gaps in the CCD chips. We perform sky subtraction and 1D spectral extraction using gsextract, which employs the task apall. Feature identification and redshift estimation are performed using the IRAF package rvsao (Kurtz \& Mink 1998).

\subsubsection{Redshift of the Lens}

The extracted un-fluxed 1D spectrum for the BCG (G1) is shown in Figure 4. The characteristic $\mathrm{Ca} \mathrm{H}$ and $\mathrm{K}$ absorption lines present in early-type galaxy spectra are visible in the spectra of all five galaxies at $\lambda=8192 \AA$ and $\lambda=8120 \AA$ respectively (indicated by the red lines in Figure 4). The redshifts obtained for the five galaxies G1-G5 using a crosscorrelation technique (Kurtz \& Mink 1998, XCSAO) are listed in Table 3. The BCG has a redshift of $z=1.0645 \pm 0.0002$. The average redshift of the five galaxies is $z=1.0644$. In addition we obtained spectra for two additional galaxies that are likely to be members of the cluster as they have very similar

\footnotetext{
${ }^{44}$ http://www.gemini.edu/sciops/data-and-results/processing-software
} 

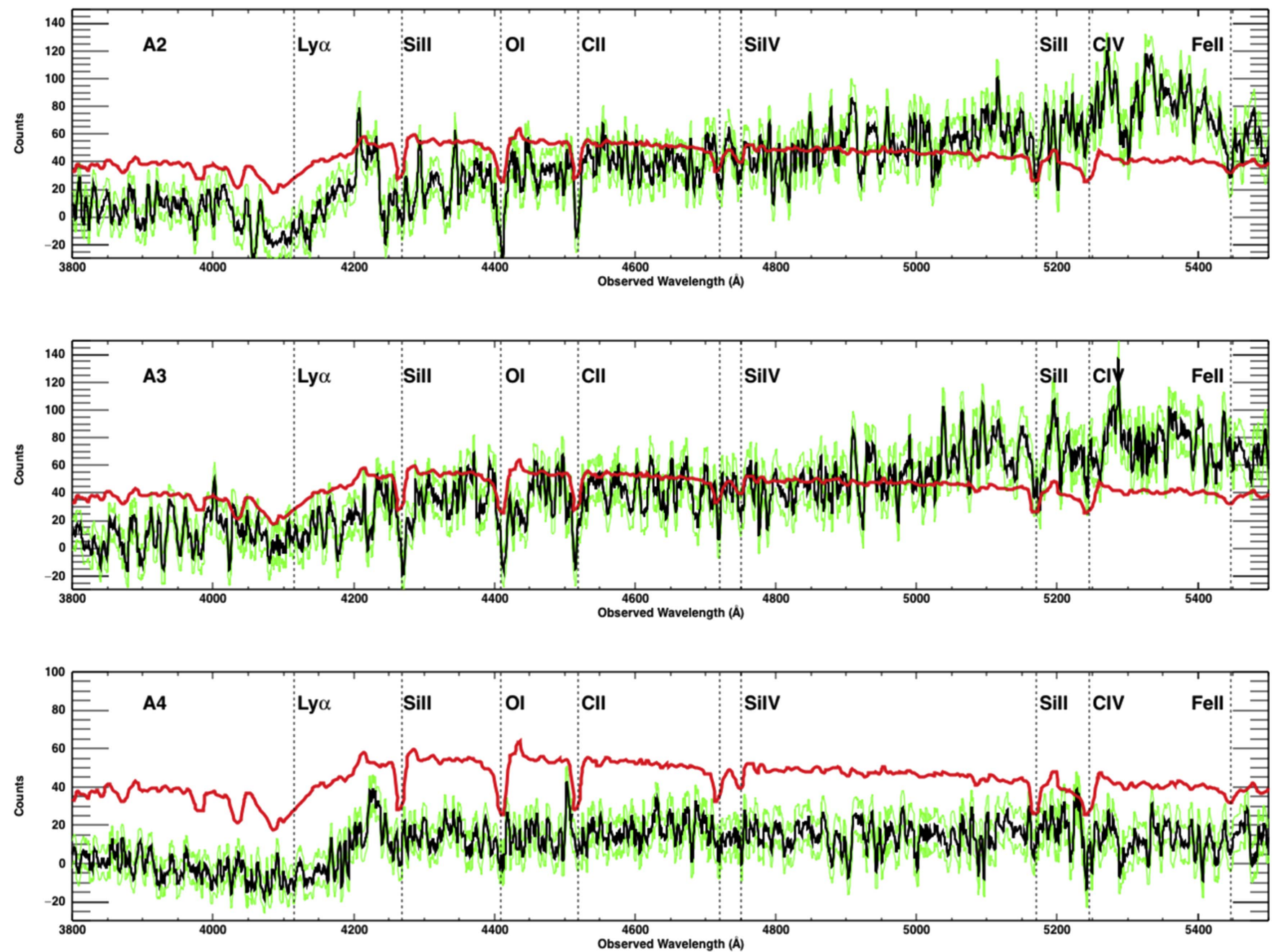

Figure 5. The extracted un-fluxed spectra for the arcs A2-A4. Absorption features from a Lyman break galaxy at $z=2.3875$ are indicated by the dotted lines. The green spectra indicate the $\pm 1 \sigma$ errors from the spectral extraction. The red spectrum is the LBG template of Shapley et al. (2003) shifted by the measured redshift.

Table 2

Redshifts for A2-A4

\begin{tabular}{lcccc}
\hline \hline Object & R.A. & Decl. & Redshift & $R$-value \\
\hline A2 & 302.777796 & -52.468769 & $2.3875 \pm 0.0002$ & 4.62 \\
A3 & 302.785149 & -52.467079 & $2.3889 \pm 0.0002$ & 5.34 \\
A4 & 302.783661 & -52.471130 & $2.3875 \pm 0.0004$ & 2.26 \\
\hline
\end{tabular}

Table 3

Redshifts for G1-G5

\begin{tabular}{lccc}
\hline \hline Object & R.A. & Decl. & Redshift \\
\hline G1 & 302.78122 & -52.47105 & $1.0645 \pm 0.0002$ \\
G2 & 302.78244 & -52.47035 & $1.0737 \pm 0.0002$ \\
G3 & 302.78418 & -52.47032 & $1.0642 \pm 0.0002$ \\
G4 & 302.78605 & -52.47087 & $1.0514 \pm 0.0002$ \\
G5 & 302.77766 & -52.46994 & $1.0684 \pm 0.0002$ \\
\hline
\end{tabular}

redshifts. They are both about 1.5 arcmin from the cluster center with redshifts of $z=1.0647$ and $z=1.0620$.

\subsubsection{Redshift of the Source}

The extracted un-fluxed spectra and their $\pm 1 \sigma$ errors (green spectra) for the arcs A2-A4 are shown in Figure 5 for the B600 grating. We detected continuum flux in all three arcs but no obvious emission line features. We do, however, observe a break in the spectrum at around $4100 \AA$ and absorption features at $4410 \AA, 4517 \AA$, and $4720 \AA$. These features are indicative of those found in the spectrum of a Lyman break galaxy (LBG) (Steidel et al. 2004) but with Ly $\alpha$ absorption rather than emission. We perform a cross-correlation (Kurtz \& Mink 1998, XCSAO) against the LBG template of Shapley et al. (2003) for all three arcs. The results are listed in Table 2. The $R$-value in Table 2 provides a measure of the confidence of the redshift extraction and the uncertainty (Tonry \& Davis 1979). The $R$-values for A2 and A3 are both greater than 4, which is an indication of a secure redshift (as defined by Kurtz \& Mink 1998). The $R$-value for A4 is lower and reflects the lower signal-to-noise ratio in the spectrum. However, the presence of a break in the spectrum at around $4100 \AA$ consistent with the ones observed in A2 and A3, and the resulting redshift, which is consistent with those obtained from A2 and A3, give us confidence that A4 is indeed a lensed image of the source galaxy. The mean redshift from the three arcs is $2.3880 \pm 0.0003$. Figure 5 shows the locations of the expected spectral features for an LBG, and the red spectrum is the LBG template of Shapley et al. (2003) shifted by the measured redshift. The redshifts of the lens and source imply a total mass within the Einstein radius of $\log _{10}\left(M / M_{\odot}\right)=14.170 \pm 0.004$. 


\section{Lens Modeling of the Lensed Arcs and Central Image}

We model J2011 with a cluster-scale dark matter halo plus subhalos to describe the baryons and dark matter associated with the cluster members and a light profile with a single Sérsic component to describe the source. The presence of the central image gives us a unique opportunity to test the central density profile of the DM halo. We therefore fit the dark matter with a generalized NFW (gNFW) profile (Wyithe et al. 2001). This allows us to test models with central cores or shallow cusps.

For the gNFW profile we assume the form

$$
\rho(r)=\frac{\rho_{0}}{r^{\alpha}\left(r_{s}^{2}+r^{2}\right)^{(3-\alpha) / 2}},
$$

where $r$ is the three-dimensional distance from the halo center. The profile is characterized by an inner slope, $\alpha$, with the density falling as $r^{\alpha}$, an outer profile slope 3 , and a break radius $r_{s}$. The case of $\alpha=1$ is approximately the NFW profile but with a slightly different behavior in the turnover region. Using $\left(r_{s}^{2}+r^{2}\right)^{1 / 2}$ in the denominator rather than $\left(r_{s}+r\right)$ has the advantage that deflection angles for the spherical model can be calculated analytically (Muñoz et al. 2001). For computational efficiency we include ellipticity in the lensing potential rather than the density profile; this is a good approximation for almost spherical halos (Barkana 1998).

In addition to the cluster-scale dark matter halo(s), mass is associated with the individual cluster members (Shaw et al. 2006). We account for this mass by placing isothermal mass clumps in the locations where cluster members are observed. Using the $z$-band data, we fit the BCG light profile with an elliptical Sérsic profile, and model the other cluster members with circular de Vaucouleurs profiles. For the BCG the ellipticity and position angle of the mass are fixed to those observed for the light profile. The other cluster members are modeled as isothermal spheres. In order to minimize the number of free parameters in the model, we assume a constant mass-to-light ratio for all the cluster members. The Einstein radius of each component is proportional to the square root of the fitted $z$-band flux of each component: the constant of proportionality, $\tilde{\Upsilon}_{\mathrm{G}}$, is a free parameter of the model; $\tilde{\Upsilon}_{\mathrm{G}}$ is scaled in units such that it is also the Einstein radius of the BCG in arcseconds. The cluster members included in our model are shown in Figure 6.

The final component of the lens model is an external shear, which allows for perturbative lensing from nearby perturbers (e.g., Holder \& Schechter 2003) and line-of-sight structures (e.g., Collett et al. 2013; McCully et al. 2017).

In order to fully exploit the information content of the central image it is necessary to attempt to reconstruct the light profiles of the lensed images. Full fitting of the light profile is more computationally challenging than conjugate point methods (as used in Jullo et al. 2007, for example), but it allows us to use the observed fluxes in many thousands of pixels as constraints on the lens model.

Full fitting of the light profile requires some assumptions to be made about the unlensed source. Simply parameterized sources are quick to calculate and are robust against predicting images where none are observed, although they often require many nonlinear source model parameters (e.g., Brewer et al. 2011), and the extra sampling negates the computational benefits. More seriously, the simplicity of the assumed light

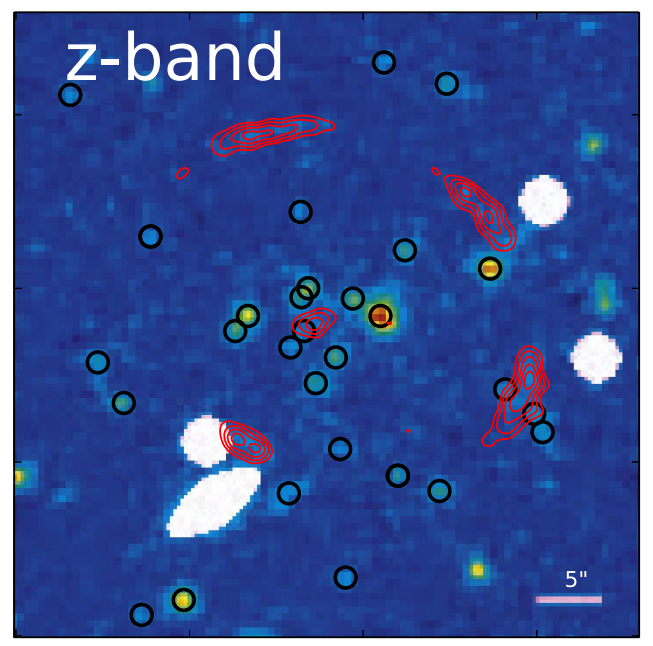

Figure 6. $z$-band image of $\mathbf{J} 2011$, with the arcs superimposed as red contours. The data have been filtered with a Gaussian filter (width 2 pixels) to pick out faint cluster members. Members included in the lens model are circled in black. The masked white regions are the locations of bright stars and a foreground galaxy.

profile means that significant residuals are often present in regions of high magnification. These residuals cause simply parameterized sources to underestimate the uncertainties on the lens model (T. E. Collett \& M. W. Auger 2017, in preparation). Pixellated sources are much more agnostic about the source profile, yielding better fits to the data and more robust estimates of the lens model parameters. However, this is only true when the lens model is reasonably close to truth: the high computational cost of pixellated source modeling means that only a small amount of data can be reconstructed - the region including and immediately surrounding the lensed imagesand lens models that predict images outside the reconstructed region are not penalized.

We therefore first optimize our models toward an approximate best fit assuming that the brightest observed pixel of each of the five lensed images are conjugate points, and use this lens model to initialize our full fitting of the light profile. We only model the parts of the image plane that contain flux from the source (shown in Figure 2). To avoid the model predicting images outside the masked region, we cast 100 pixels (outside the mask) back onto the source plane and discard any model for which these pixels are within the source, defined as the smallest circle containing all five conjugate points. For the best fit model we also remodel the system with a much larger mask and verify visually that there are no extra images predicted.

For the source profile we adopt a pixellated source model, following the semilinear approach of Warren \& Dye (2003). We use an adaptive 50 by 50 grid of square pixels as detailed in Collett \& Auger (2014) in order to avoid artificially breaking the mass-sheet degeneracy (Falco et al. 1985). We use curvature-regularized sources as favored by the analysis of Suyu et al. (2006); the regularization encodes the prior that astrophysical sources are reasonably smooth. We follow the Bayesian prescription of Suyu et al. (2006) and allow the data to tell us the optimal degree of source regularization for each iteration of the lens model.

Our lens model now has 10 free nonlinear parameters: two for the centroid of the halo $\left(x_{\text {lens }}, y_{\text {lens }}\right)$, one for the Einstein radius of the main halo $\left(\theta_{\mathrm{E}}\right)$, two for the ellipticity and position 
Data

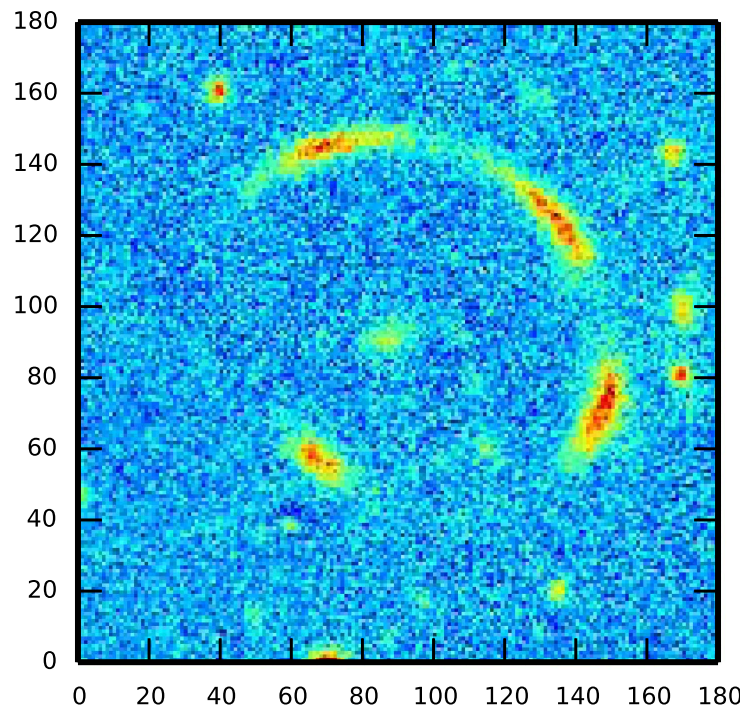

Residual

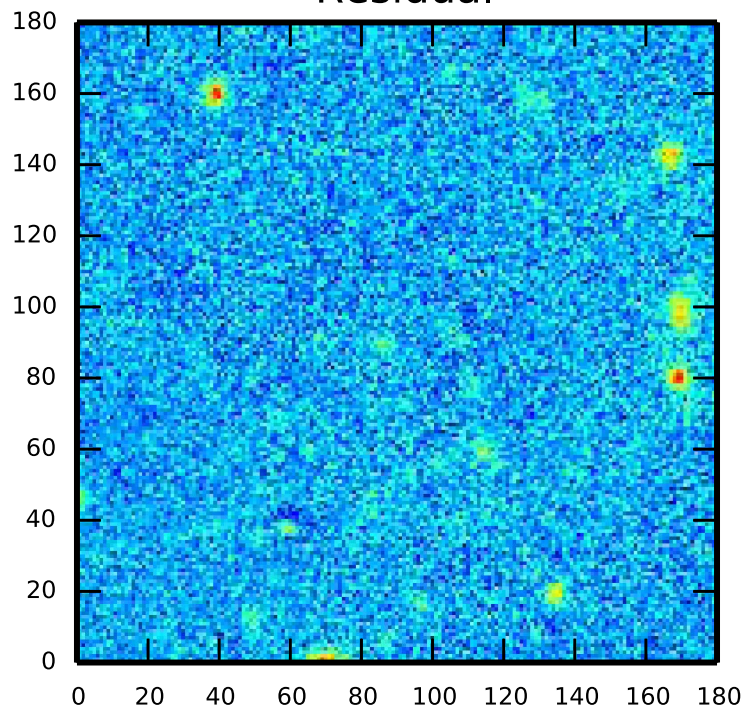

Model

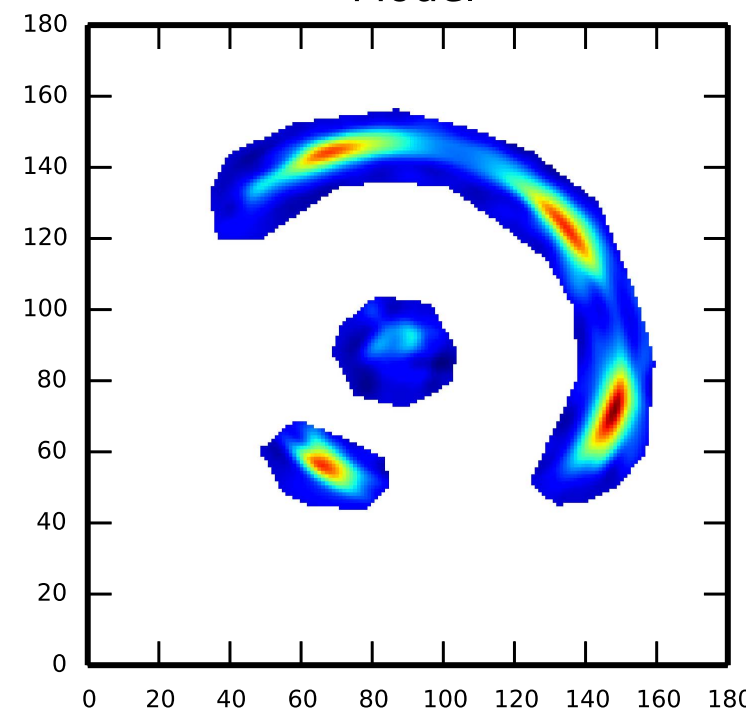

Source

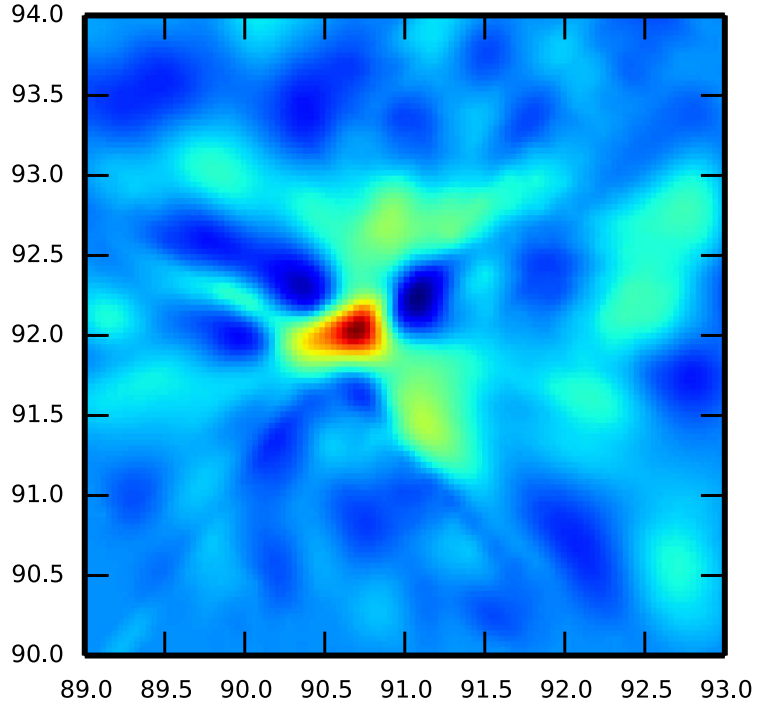

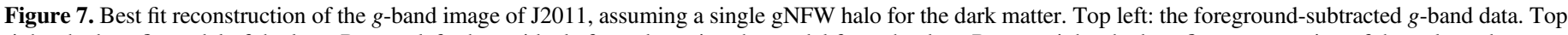

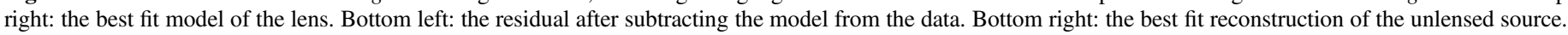
0,0 is at 20:11:10.611, $-52: 28: 40.12$ (J2000) with a pixel size of 0.263 arcsec.

Table 4

The Inferred Parameters for the One-halo Model

\begin{tabular}{|c|c|c|c|c|c|c|c|c|c|c|}
\hline Parameter & $x_{\text {lens }}$ & $y_{\text {lens }}$ & $\theta_{\mathrm{E}}$ & $q_{\text {lens }}$ & $\overline{\theta_{q}}$ & $\bar{\alpha} \alpha$ & $r_{\mathrm{s}}(\mathrm{kpc})$ & $\overline{\gamma \gamma}$ & 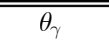 & $\overline{\overline{\Upsilon_{\mathrm{G}}}}$ \\
\hline Inference & $90.6_{-0.3}^{+0.2}$ & $91.6_{-0.2}^{+0.2}$ & $13.6_{-0.4}^{+0.3}$ & $0.866_{-0.007}^{+0.006}$ & $153_{-3}^{+3}$ & $0.38_{-0.04}^{+0.04}$ & $277_{-74}^{+93}$ & $0.081_{-0.004}^{+0.006}$ & $61.7_{-1.6}^{+2.2}$ & $0.02_{-0.02}^{+0.02}$ \\
\hline
\end{tabular}

angle of the halo $\left(q_{\text {lens }}, \theta_{q}\right)$, the inner slope $(\alpha)$ and break radius of the halo $\left(r_{\mathrm{s}}\right)$, two parameters for the external shear $\left(\gamma, \theta_{\gamma}\right)$, and one for the mass-to-light ratio of the cluster members $\left(\tilde{\Upsilon}_{\mathrm{G}}\right)$. We probe the posterior of these nonlinear parameters using a Markov chain Monte Carlo (MCMC) method. We use the ensemble sampler of Foreman-Mackey et al. (2013).

\subsection{Results: A Shallow Cusp in the Dark Matter Halo}

Applying the model of the previous section to J2011, we find that the DES data can be well reconstructed with an astrophysically reasonable source and a plausible mass distribution for the lens. The best fit reconstruction of the data is shown in Figure 7. Our inference on the model has an almost spherical dark matter halo with flattening $q=0.87$, a shallow inner density slope, $\rho \sim r^{-0.35}$, and a scale radius of $290 \mathrm{kpc}$; $2.2 \%$ of the mass within the Einstein radius is in subhalos. We see that the arcs and central image are reproduced with surprisingly small residuals given the simplicity of the mass model. The model slightly underpredicts the flux of the central image, indicating that the true central profile may be shallower than quoted. Due to the 1 arcsec seeing of our imaging, the 


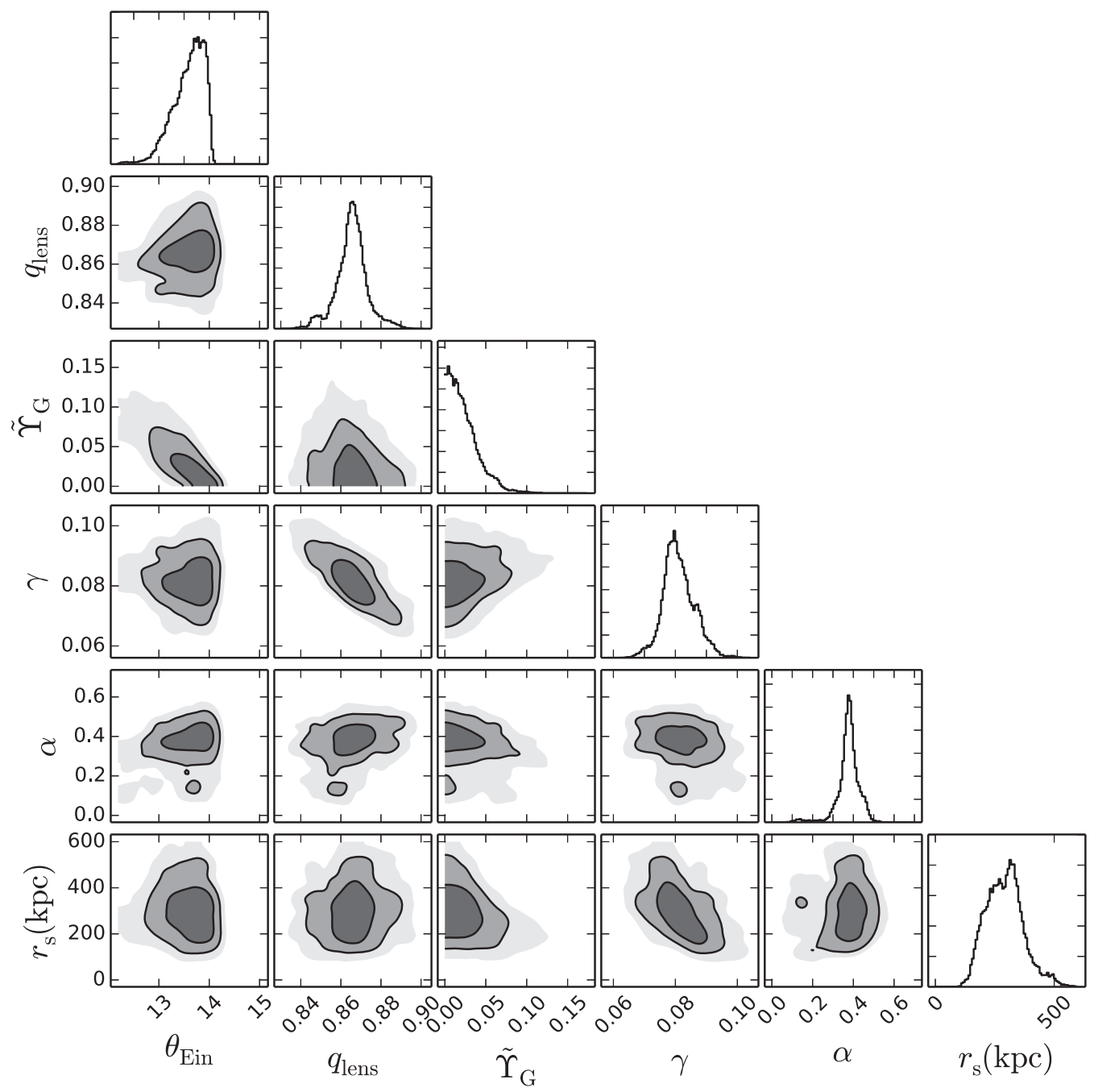

Figure 8. Marginalized one- and two-dimensional parameter constraints for the model of J2011 with a single dark matter halo. The contours show the $68 \%$, $95 \%$, and $99 \%$ confidence regions. $\theta_{\mathrm{E}}$ is the Einstein radius of the DM halo (not the total Einstein radius of the arcs, since this is made up from the DM and the cluster members). The flattening of the DM halo is $q_{\text {lens }}, \alpha$ is the inner profile slope, and $r_{\mathrm{s}}(\mathrm{kpc})$ is the scale radius of the gNFW halo. $\tilde{\Upsilon}_{\mathrm{G}}$ relates the observed $z$-band fluxes and the Einstein radius of the cluster members; it is in units such that $\tilde{\Upsilon}_{\mathrm{G}}$ is the Einstein radius of the BCG in arcseconds. The inner profile slope and the scale radius of the gNFW halo have only a mild covariance with the unshown parameters of the model.

reconstructed source is not well resolved; however, the model predicts that the source has one bright clump and possibly two extended nearby features. This irregularity is typical of the blue sources reconstructed in previous strong lensing studies (e.g., Brewer et al. 2011; Shu et al. 2016).

Our MCMC results show that the DM halo requires a shallow central density profile. The one-dimensional parameter constraints are shown in Table 4. Figure 8 shows the marginalized twodimensional posterior for the DM halo properties. The inner slope and scale radius are degenerate, but inner slopes steeper than $r^{-0.55}$ and scale radii smaller than $100 \mathrm{kpc}$ are strongly excluded, indicating that the central profile deviates from the NFW prediction of $r^{-1}$ over a large radius. Marginalizing over the other parameters, we derive $68 \%$ confidence intervals of $\alpha=0.38 \pm 0.04$ and $r_{s}=244_{-64}^{+81} \mathrm{kpc}$. The halo flattening is constrained to be $q_{\text {lens }}=0.877_{-0.007}^{+0.006}$. The external shear is $\gamma=0.083 \pm 0.005$. And the constant of proportionality relating $z$-band flux to cluster member mass is $\tilde{\Upsilon}_{\mathrm{G}}=0.02_{-0.02}^{+0.02}$, defined in units where $\tilde{\Upsilon}_{\mathrm{G}}$ is the Einstein radius of the BCG in arcseconds, and the Einstein radii of the other members vary as $\theta_{i}=\theta_{\mathrm{BCG}}\left(z_{i}^{\text {Flux }} / z_{\mathrm{BCG}}^{\text {Flux }}\right)^{1 / 2}$.

\section{An Alternative Model: Two Merging Dark Matter Halos}

In the previous section we found that the arcs and central image cannot be adequately reconstructed by a single (non-generalized) NFW halo, but the gNFW gets very close to reproducing the lens system, despite having only a small number of free parameters.

However, the fact that the gNFW model requires a much shallower profile than simulations predict and the slight underfitting of the central image imply that the true mass distribution may be more complicated than our simple gNFW model allows. One alternative hypothesis is that J2011 is a merger of two (or more) subhalos. We test this theory by adding a second gNFW clump to the model used in the previous section. Since the halos are no longer guaranteed to be critical, we characterize them by their central convergence, $\kappa_{s, i}$, as defined in Equation (3) of Muñoz et al. (2001) and not by their Einstein radius. We also do not include external shear in the two-halo model because it is not required to reproduce the data.

The two-halo model is also able to reconstruct the data: our best-fit two-halo model is shown in Figure 9. The two-halo 
Data
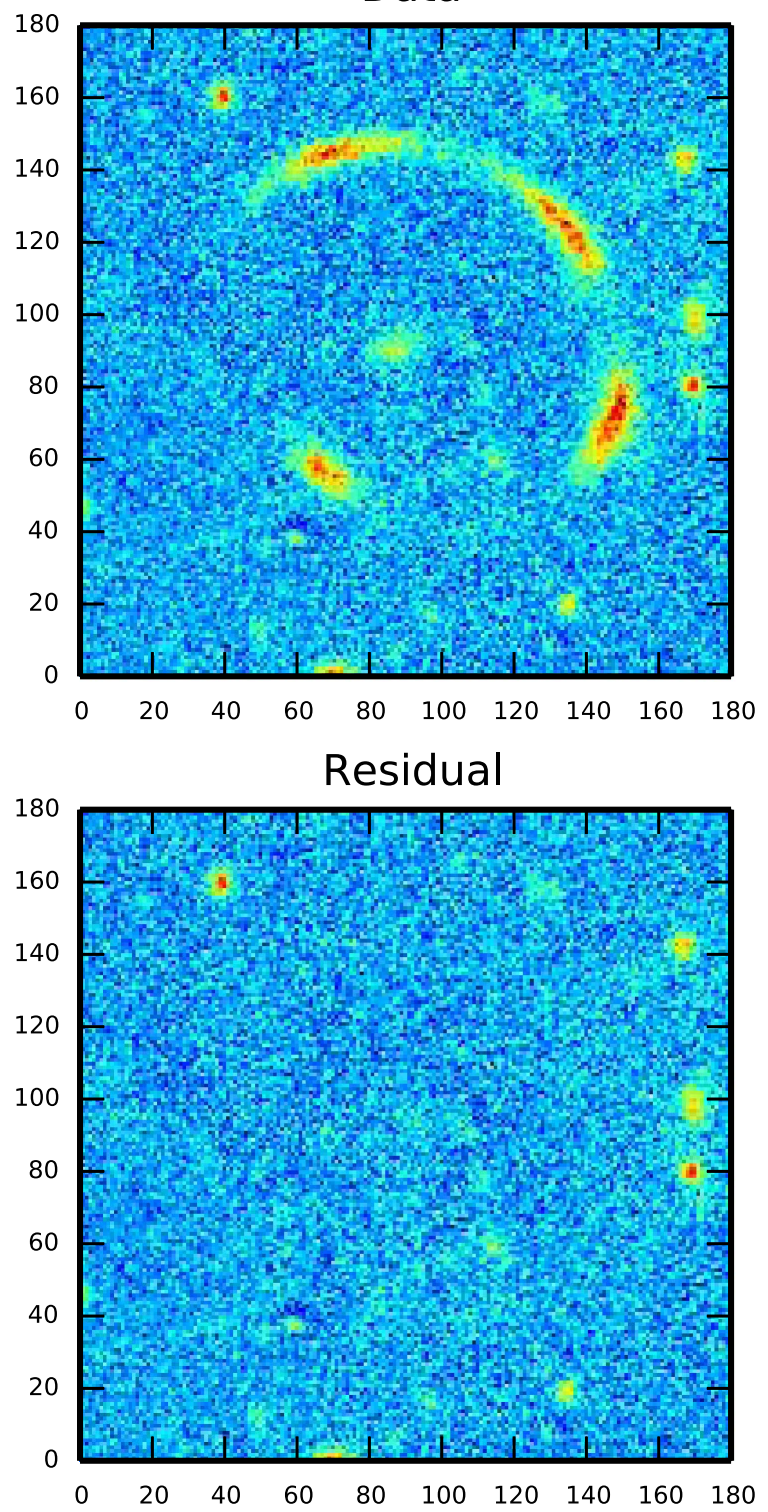

Model
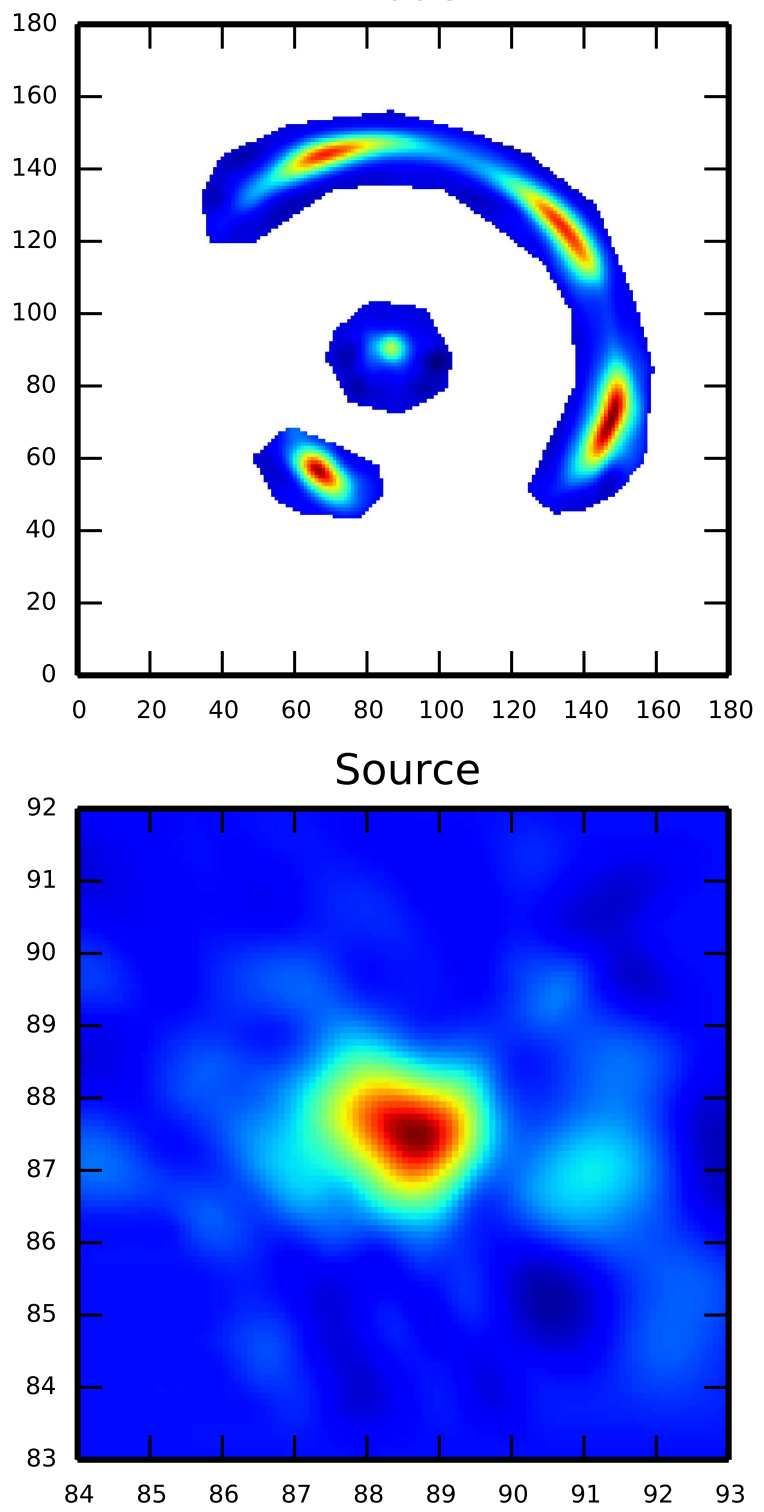

Figure 9. Best fit reconstruction of J2011. The same as Figure 7, but assuming a model with two dark matter halos for the lensing mass.

Table 5

The Inferred Parameters for the Two-halo Model

\begin{tabular}{|c|c|c|c|c|c|c|c|c|}
\hline Parameter & $x_{\text {lens, } 1}$ & $y_{\text {lens, } 1}$ & $\kappa_{s, 1}$ & $q_{\text {lens }, 1}$ & $\theta_{q, 1}$ & $\alpha_{1}$ & $r_{\mathrm{s}, 1}(\mathrm{kpc})$ & $\Upsilon_{\mathrm{G}}$ \\
\hline Inference & $82.9_{-0.5}^{+0.5}$ & $94.4_{-0.7}^{+0.7}$ & $0.032_{-0.005}^{+0.004}$ & $0.90_{-0.02}^{+0.02}$ & $-49.6_{-4.3}^{+3.6}$ & $0.98_{-0.05}^{+0.08}$ & $395_{-54}^{+53}$ & $0.55_{-0.06}^{+0.08}$ \\
\hline Parameter & $x_{\text {lens }, 2}$ & $y_{\text {lens }, 2}$ & $\kappa_{s, 2}$ & $q_{\text {lens }, 2}$ & $\theta_{q, 2}$ & $\alpha_{2}$ & $r_{\mathrm{s}, 2}(\mathrm{kpc})$ & \\
\hline Inference & $91.3_{-1.6}^{+1.5}$ & $73.3_{-2.4}^{+2.1}$ & $0.07_{-0.01}^{+0.01}$ & $0.52_{-0.04}^{+0.04}$ & $48.2_{-0.9}^{+0.8}$ & $0.79_{-0.08}^{+0.09}$ & $433_{-46}^{+57}$ & \\
\hline
\end{tabular}

model parameters are summarized in Table 5 and Figure 10. This model also prefers both halos to be approximately NFW. The model requires most of the mass to be in an almost spherical NFW halo, to the northwest of the central image, with a less massive, highly flattened component to the southeast. In this model $20 \%$ of the mass within the Einstein radius is associated with subhalos. The central density of the two halos varies as radius to the powers $0.99_{-0.06}^{+0.08}$ and $0.79 \pm 0.09$ respectively. The break radii are $345_{-42}^{+48} \mathrm{kpc}$ and $48.9_{-5.6}^{+6.6} \mathrm{kpc}$. We show the convergence map of the two dark matter halos in Figure 11.
Figure 12 shows the convergence of the total density profile for various slices through the central image. Despite the fact that the DM halos and the cluster members are cuspy, the sum of the components gives a remarkably flat profile at the location of the central image.

\section{Interpretation of J2011: A Tension with $\Lambda \mathrm{CDM}$ or a Merger of Two NFW Halos?}

Both the one- and two-halo models are able to reproduce the observed light profile of the arcs and the central image. The 


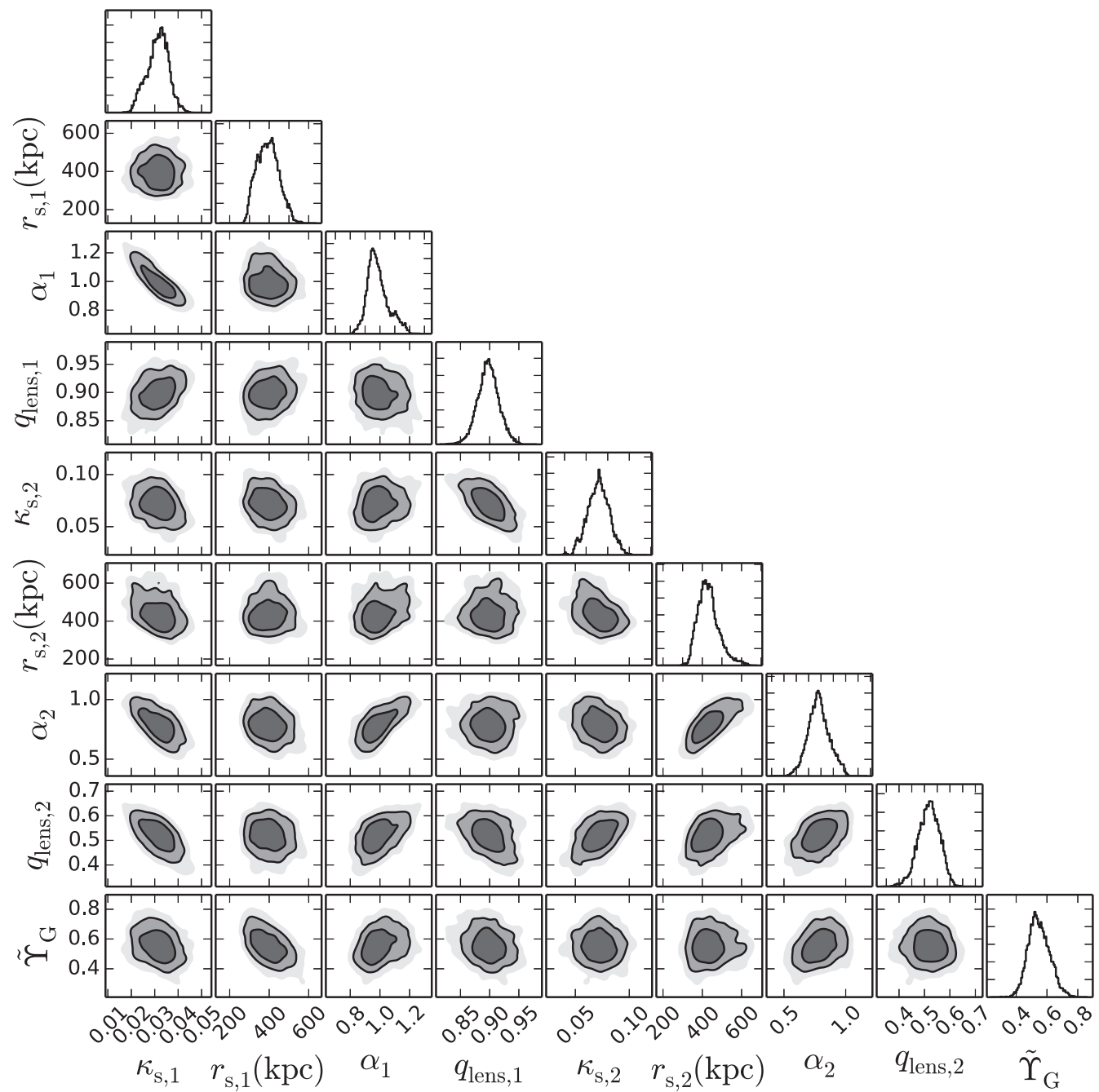

Figure 10. Marginalized one- and two-dimensional parameter constraints for the model of J2011 with two dark matter halos. The contours show the $68 \%$, 95\%, and 99\% confidence regions. Parameters with subscript 1 refer to the primary clump and those with subscript 2 refer to the secondary clump.

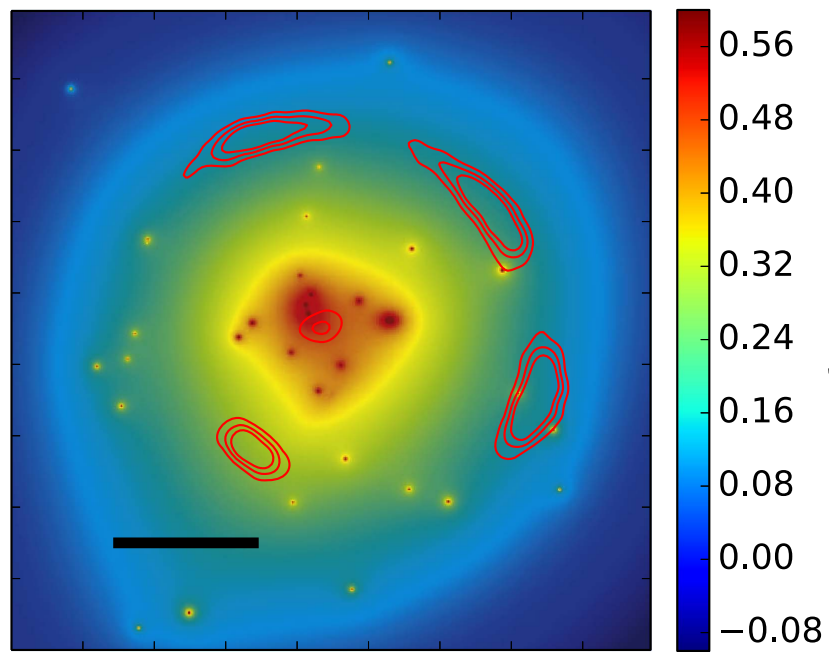

Figure 11. Mass distribution in J2011 as inferred from the model with two DM halos. The red contours show the location of the lensed images. The black ellipses indicate the locations and flattenings of the two DM halos. The black bar indicates a scale of 10 arcsec.

two-halo model provides a somewhat better fit to the data. However, the log likelihoods (base 10) of the residuals differ by only 17 totalled over 4892 pixels. The two-halo model has five extra free parameters but also has a comparatively simpler source: the penalization term of the merit function (Suyu et al. 2006) prefers the two-halo source by 19 . Without the source penalization term, the Bayesian information criterion (Schwarz 1978) would prefer the one-halo model, but with the penalization term it prefers the two-halo model.

The source penalization term of Suyu et al. (2006) is designed, however, to prefer smoother sources, rather than to precisely encode a known astrophysical prior about the clumpiness of sources. It is therefore unclear how to use the regularization term in quantitative selection of a Bayesian model. This same issue has been tackled in time delay cosmography (Wong et al. 2016), with similarly unsatisfactory conclusions. We therefore conclude that with our data alone, both models are reasonable interpretations for this system.

The choice between the two models thus reverts to astrophysical priors. From a physical perspective, neither model is totally satisfactory: in neither model is a DM halo centered on the BCG. For the one-halo model the DM centroid is offset from the BCG by $30 \mathrm{kpc}$. For the two-halo model the main DM halo is centered around the clump of galaxies to the northwest of the central image. The second DM halo is associated with the pair of galaxies to the south-although given the extreme elongation of this halo it may be more likely that these galaxies are associated 


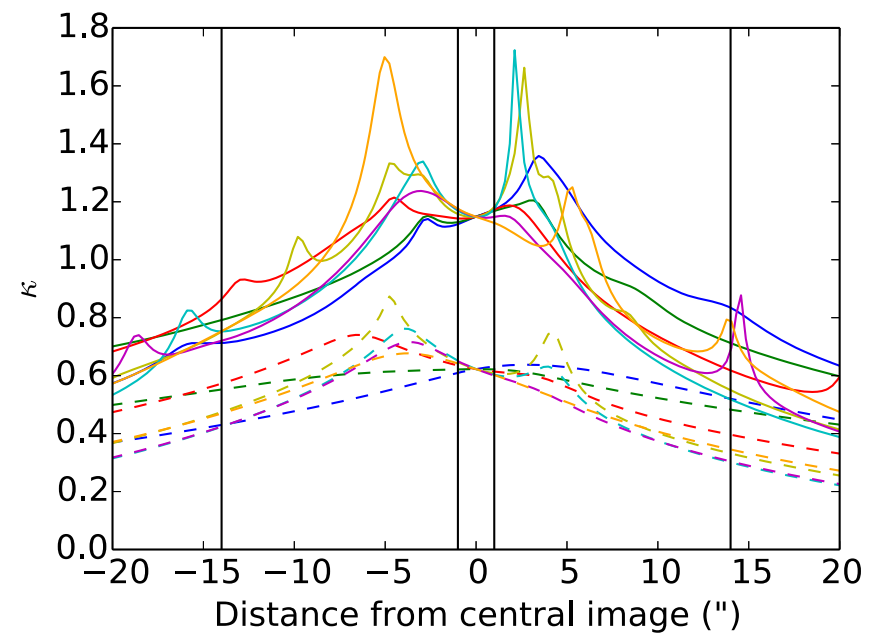

Figure 12. One-dimensional slices through the surface mass distribution of J2011. Each slice goes through the central image, but the angles of the slices vary. The solid lines show the total surface mass distribution; the dashed lines show only the contribution from the inferred DM halos. The outer vertical lines indicate the Einstein radius. The inner vertical lines indicate the approximate size of the central image.

with two separate DM halos. The offset between the DM and the BCG in the one-halo model is physically unrealistic in $\Lambda \mathrm{CDM}$ (Schaller et al. 2015); however, since $\Lambda$ CDM should also not create giant dark matter cores, the two problems may be linked. In the two-halo scenario it may not be surprising to see an offset between DM and the BCG because the system is not relaxed. The small Einstein radii of the cluster members in the one-halo model are also in tension with the typical Einstein radii of galaxy-scale lenses at lower redshifts. The one-halo model implies a maximum velocity dispersion of only $60 \mathrm{~km} \mathrm{~s}^{-1}$ for the BCG while the twohalo model gives a velocity dispersion of $219 \pm 15 \mathrm{~km} \mathrm{~s}^{-1}$. This may rule out the one-halo model, but it could also be due to our simplistic assumptions about the density profiles of the cluster members.

Both our models assume a single lens plane, neglecting lensing by mass along the line of sight (e.g., McCully et al. 2017). There is a foreground spiral galaxy centered 5 arcsec southeast of Arc 5, which we have neglected throughout this work, although it may contribute to the external shear of the one-halo model. There is no evidence that it significantly modifies the lensing potential at Arc 5, but even small changes in the geometry of a compound lens can have a significant effect in rare cases (Collett \& Bacon 2016).

Conclusively discriminating between the two models presented in this work will require higher resolution imaging. Hubble Space Telescope (HST) program GO 14630 (PI: Collett) will observe J2011 in cycle 24-and will trivially distinguish between the two models presented in this work. ${ }^{45}$ We show simulated HST images for this program in Figure 13. The figures are generated assuming the sources are no more clumpy than those reconstructed from the low-resolution DES data.

It is clear from Figure 13 that while both models yield similar images in the DES data, the predictions for the HST data are very different, with both the shape of the central image

\footnotetext{
${ }^{45}$ The $H S T$ data were taken shortly before submission of this paper. To avoid confirmation bias none of the authors of this paper (including the PI) had data access rights to view the $H S T$ data before this paper was submitted.
}
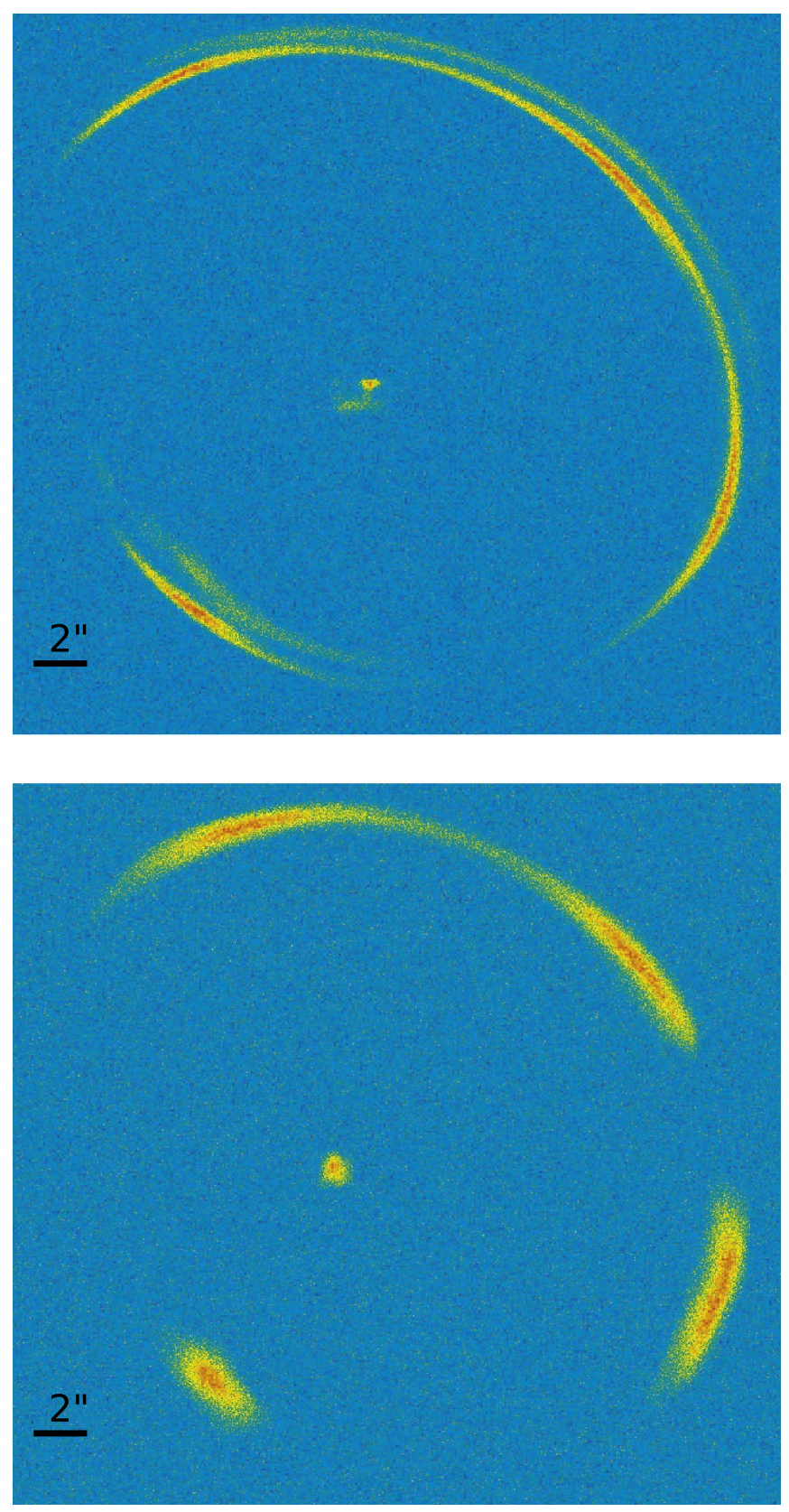

Figure 13. Simulated HST observations of J2011. The top image shows the expected $H S T$ image for the model with a single dark matter halo; the bottom image is for the two-halo model. Noise levels are those expected for HST program GO 14630 (PI: Collett) scheduled for observations in cycle 24.

and the radial width of the arcs providing tight constraints on the dark matter distribution.

\section{Conclusions}

In this work we have presented SPT-CLJ2011-5228, a giant system of arcs created by a cluster at $z=1$ possessing a unique central image. The cluster was already known from the SPT (Song et al. 2012), but we have added follow-up in the form of DES imaging and Gemini spectroscopy.

With our data we have been able to spectroscopically confirm the cluster redshift to be $z_{l}=1.06$. The source is a Lyman break galaxy at $z=2.39$, implying a total mass within 
the $\operatorname{arc}$ of $\log _{10}\left(M / M_{\odot}\right)=14.169 \pm 0.004$, consistent with the SZ mass of $M_{500}=(2.59 \pm 0.73) \times 10^{14} h_{70}^{-1} M_{\odot} \quad($ Bleem et al. 2015).

Irrespective of the source redshift we are able to model the density profile of the cluster. By describing the cluster as a single gNFW halo plus isothermal cluster members, we are able to reconstruct the arcs and central image so long as the DM halo is significantly shallower than NFW within the central $270_{-76}^{+48} \mathrm{kpc}$. We have also presented a two-halo model that reproduces the images. In this model both halos are cuspy, but the total profile at the location of the central image is remarkably flat, allowing for the formation of a bright central image that is not demagnified.

At the signal-to-noise ratio and resolution and of our imaging, the merger (two-halo) model provides a somewhat better fit to the data than the shallow cusped (one-halo) model, but at the expense of extra free parameters. Forthcoming HST imaging will be able to conclusively resolve between the shallow cusp and merger models. The stakes for these data are high, because the one-halo model cannot be consistent with the concordance model of cold dark matter unless extreme amounts of baryonic feedback have resculpted the halo over hundreds of kiloparsecs. The forthcoming HST data will soon allow us to explain the unique central image of J2011, infer the dark matter profile in this system, and perhaps shine a light on the nature of dark matter.

We thank the referee for their helpful comments in improving upon the original manuscript. We are grateful to Tomasso Treu and Wojciech Hellwing for fruitful discussions. T.E.C. is grateful to Matt Auger for his significant contributions to the lens modeling code used in this work. We are grateful to Marusa Bradac and Matt Auger for encouraging us to investigate the merging halo scenario.

Funding for the DES Projects has been provided by the U.S. Department of Energy, the U.S. National Science Foundation, the Ministry of Science and Education of Spain, the Science and Technology Facilities Council of the United Kingdom, the Higher Education Funding Council for England, the National Center for Supercomputing Applications at the University of Illinois at Urbana-Champaign, the Kavli Institute of Cosmological Physics at the University of Chicago, the Center for Cosmology and Astro-Particle Physics at the Ohio State University, the Mitchell Institute for Fundamental Physics and Astronomy at Texas A\&M University, Financiadora de Estudos e Projetos, Fundação Carlos Chagas Filho de Amparo à Pesquisa do Estado do Rio de Janeiro, Conselho Nacional de Desenvolvimento Científico e Tecnológico and the Ministério da Ciência, Tecnologia e Inovação, the Deutsche Forschungsgemeinschaft, and the Collaborating Institutions in the Dark Energy Survey.

The Collaborating Institutions are Argonne National Laboratory, the University of California at Santa Cruz, the University of Cambridge, Centro de Investigaciones Energéticas, Medioambientales y Tecnológicas-Madrid, the University of Chicago, University College London, the DES-Brazil Consortium, the University of Edinburgh, the Eidgenössische Technische Hochschule (ETH) Zürich, Fermi National Accelerator Laboratory, the University of Illinois at Urbana-Champaign, the Institut de Ciències de l'Espai (IEEC/CSIC), the Institut de Física d'Altes Energies, Lawrence Berkeley National Laboratory, the Ludwig-Maximilians Universität München and the associated Excellence Cluster Universe, the University of Michigan, the National Optical Astronomy Observatory, the University of Nottingham, The Ohio State University, the
University of Pennsylvania, the University of Portsmouth, SLAC National Accelerator Laboratory, Stanford University, the University of Sussex, Texas A\&M University, and the OzDES Membership Consortium.

The DES data management system is supported by the National Science Foundation under Grant Number AST-1138766. The DES participants from Spanish institutions are partially supported by MINECO under grants AYA2015-71825, ESP2015-88861, FPA2015-68048, SEV-2012-0234, SEV-2012-0249, and MDM2015-0509, some of which include ERDF funds from the European Union. IFAE is partially funded by the CERCA program of the Generalitat de Catalunya.

Based on observations obtained at the Gemini Observatory (processed using the Gemini IRAF package), which is operated by the Association of Universities for Research in Astronomy, Inc., under a cooperative agreement with the NSF on behalf of the Gemini partnership: the National Science Foundation (United States), the National Research Council (Canada), CONICYT (Chile), Ministerio de Ciencia, Tecnología e Innovación Productiva (Argentina), and Ministério da Ciência, Tecnologia e Inovação (Brazil).

\section{References}

Abadi, M. G., Navarro, J. F., Fardal, M., Babul, A., \& Steinmetz, M. 2010, MNRAS, 407, 435

Amorisco, N. C., Agnello, A., \& Evans, N. W. 2013, MNRAS, 429, L89

Balbinot, E., Santiago, B. X., Girardi, L., et al. 2015, MNRAS, 449, 1129

Barkana, R. 1998, ApJ, 502, 531

Benítez, N. 2000, ApJ, 536, 571

Bertin, E. 2011, ASPC, 442, 435

Bertin, E., \& Arnouts, S. 1996, A\&AS, 117, 393

Bleem, L. E., Stalder, B., de Haan, T., et al. 2015, ApJS, 216, 27

Brewer, B. J., Lewis, G. F., Belokurov, V., et al. 2011, MNRAS, 412, 2521

Coe, D., Benítez, N., Sánchez, S. F., et al. 2006, AJ, 132, 926

Collett, T. E., \& Auger, M. W. 2014, MNRAS, 443, 969

Collett, T. E., \& Bacon, D. J. 2016, MNRAS, 456, 2210

Collett, T. E., Marshall, P. J., Auger, M. W., et al. 2013, MNRAS, 432, 679

Colley, W. N., Tyson, J. A., \& Turner, E. L. 1996, ApJL, 461, L83

de Blok, W. J. G., McGaugh, S. S., \& Rubin, V. C. 2001, AJ, 122, 2396

Desai, S., Armstrong, R., Mohr, J. J., et al. 2012, ApJ, 757, 83

Diehl, H. T., Abbott, T. M. C., Annis, J., et al. 2014, Proc. SPIE, 9149, 91490V

Duffy, A. R., Schaye, J., Kay, S. T., et al. 2010, MNRAS, 405, 2161

Falco, E. E., Gorenstein, M. V., \& Shapiro, I. I. 1985, ApJL, 289, L1

Flaugher, B. 2005, IJMPA, 20, 3121

Flaugher, B., Diehl, H. T., Honscheid, K., et al. 2015, AJ, 150, 150

Foreman-Mackey, D., Hogg, D. W., Lang, D., \& Goodman, J. 2013, PASP, 125,306

Gnedin, O. Y., Kravtsov, A. V., Klypin, A. A., \& Nagai, D. 2004, ApJ, 616, 16 Grillo, C. 2012, ApJL, 747, L15

Holder, G. P., \& Schechter, P. L. 2003, ApJ, 589, 688

Hook, I. M., Jørgensen, I., Allington-Smith, J. R., et al. 2004, PASP, 116, 425

Inada, N., Oguri, M., Keeton, C. R., et al. 2005, PASJ, 57, L7

Jullo, E., Kneib, J.-P., Limousin, M., et al. 2007, NJPh, 9, 447

Kurtz, M. J., \& Mink, D. J. 1998, PASP, 110, 934

Laporte, C. F. P., White, S. D. M., Naab, T., Ruszkowski, M., \& Springel, V. 2012, MNRAS, 424, 747

McCully, C., Keeton, C. R., Wong, K. C., \& Zabludoff, A. I. 2017, ApJ, 836, 141

Mohr, J. J., Armstrong, R., Bertin, E., et al. 2012, Proc. SPIE, 8451, 84510D

Muñoz, J. A., Kochanek, C. S., \& Keeton, C. R. 2001, ApJ, 558, 657

Navarro, J. F., Frenk, C. S., \& White, S. D. M. 1996, ApJ, 462, 563

Neto, A. F., Gao, L., Bett, P., et al. 2007, MNRAS, 381, 1450

Newman, A. B., Treu, T., Ellis, R. S., et al. 2013, ApJ, 765, 24

Newman, A. B., Treu, T., Ellis, R. S., \& Sand, D. J. 2013, ApJ, 765, 25

Oldham, L. J., \& Auger, M. W. 2016, MNRAS, 457, 421

Peter, A. H. G., Rocha, M., Bullock, J. S., \& Kaplinghat, M. 2013, MNRAS, 430, 105

Pineda, J. C. B., Hayward, C. C., Springel, V., \& Mendes de Oliveira, C. 2017, MNRAS, 466, 63

Planck Collaboration, Ade, P. A. R., Aghanim, N., et al. 2016, A\&A, 594, A13

Pontzen, A., \& Governato, F. 2012, MNRAS, 421, 3464 
Reichardt, C. L., Stalder, B., Bleem, L. E., et al. 2013, ApJ, 763, 127

Rocha, M., Peter, A. H. G., Bullock, J. S., et al. 2013, MNRAS, 430, 81

Sand, D. J., Treu, T., Smith, G. P., \& Ellis, R. S. 2004, ApJ, 604, 88

Schaller, M., Robertson, A., Massey, R., Bower, R. G., \& Eke, V. R. 2015, MNRAS, 453, L58

Schwarz, G. E. 1978, AnSta, 6, 461

Scoville, N., Aussel, H., Brusa, M., et al. 2007, ApJS, 172, 1

Sevilla, I., Armstrong, R., Bertin, E., et al. 2011, arXiv:1109.6741

Shapley, A., Steidel, C., Pettini, M., \& Adelberger, K. L. 2003, AJ, 588, 65

Sharon, K., Gladders, M. D., Rigby, J. R., et al. 2012, ApJ, 746, 161

Shaw, L. D., Weller, J., Ostriker, J. P., \& Bode, P. 2006, ApJ, 646, 815

Shu, Y., Bolton, A. S., Mao, S., et al. 2016, ApJ, 833, 264

Song, J., Zenteno, A., Stalder, B., et al. 2012, ApJ, 761, 22
Sonnenfeld, A., Treu, T., Gavazzi, R., et al. 2012, ApJ, 752, 163

Steidel, C., Shapley, A. E., Pettini, M., et al. 2004, ApJ, 604, 534

Suyu, S. H., Marshall, P. J., Hobson, M. P., \& Blandford, R. D. 2006, MNRAS, 371, 983

Teyssier, R., Moore, B., Martizzi, D., Dubois, Y., \& Mayer, L. 2011, MNRAS, 414, 195

Tonry, J., \& Davis, M. 1979, AJ, 84, 1511

Vogelsberger, M., Zavala, J., \& Loeb, A. 2012, MNRAS, 423, 3740

Warren, S. J., \& Dye, S. 2003, ApJ, 590, 673

Winn, J. N., Rusin, D., \& Kochanek, C. S. 2004, Natur, 427, 613

Wong, K. C., Suyu, S. H., Auger, M. W., et al. 2017, MNRAS, 465, 4895

Wyithe, J. S. B., Turner, E. L., \& Spergel, D. N. 2001, ApJ, 555, 504 